\title{
ATM Interworking Unit with ISDN and Ethernet Interfaces
}

\author{
Joaquim Neves Member, IEEE
}

\begin{abstract}
The convergence of telecommunications and computer communication technologies requires the development of Interworking Units to convert different data formats, protocols and control procedures. This paper describes the architecture of an electronic device, which is able to perform interworking functionalities between ATM, ISDN and Ethernet networks and presents several scenarios for interconnection environments.
\end{abstract}

Index terms-- ATM, ISDN, Ethernet, LANE, Interworking.

\section{INTRODUCTION}

Digital communications environments are generally characterised by two main support technologies. By one side, on computer oriented communications, Local Area Networks (LAN), supported by Ethernet or Token Ring specifications, based on data packets, are widely used to interconnect locally or remotely computers and terminals. By other side, Public Switched Networks (PSN), based on data circuits, such as the Integrated Services Digital Networks (ISDN), have not only capabilities to interconnect a large variety of terminal equipment, but also to interconnect remotely several LANs, through switched or permanent constant rate links.

The introduction of the Asynchronous Transfer Mode (ATM), which is neither circuit nor packet oriented transmission protocol but a conjunction of the best of the both techniques, permits the convergence of routing and switching technologies and the specification of new communication platforms, supported by different interfaces and transfer protocols.

The integration of this emergent technology, within telecommunication and computer networks, requests the development of special adaptation units with interworking functionalities. The compatibility between different types of services requires the development of adaptation protocols for data presentation, at the application level, namely for non-ATM, narrow or broad band services. On the other end, the compatibility between distinct systems forces the

Joaquim.Neves@dei.uminho.pt

Phone: +351253510190 Fax: +351253510189

DEI - Escola de Engenharia, Universidade do Minho

Campus de Azurém, P-4800-058 Guimarães, Portugal specification of in common physical and logical interfaces and the development of Interworking Units (IWU), to adapt different transmission, switch and flow control protocols.

This paper presents the architecture of an electronic device, which is able to handle the interworking functionalities between ISDN, ATM and Ethernet environments, and discusses the integration of such device on different network scenarios. On the next section, it is presented the architecture of the Interworking Unit while the section three characterizes the main interfaces of the system. Section four describes some interconnection scenarios and the section five summarizes the main conclusions.

\section{SyStem ARChiteCTURE}

Since the main objective of the ATM Interworking Unit is the conversion of ISDN and Ethernet traffic to the ATM formats, most conversion procedures have to be located on lower layers of the OSI protocol reference model, namely the Physical Layer and the Medium Access Control Layer. On the upper layers, the information flow generated by network applications can be carried out transparently, through the IWU, over different transmission or network protocols such as IP, IPX, NetBEUI, AppleTalk or DECNet.

The figure 1 illustrates the interconnection of Ethernet, ISDN and ATM terminals through the Interworking Unit. The hardware architecture of the proposed IWU is based on a central processor, which manages the data transfer between different line interface modules and the memory, as is sketched on the bloc diagram of the figure 2 .

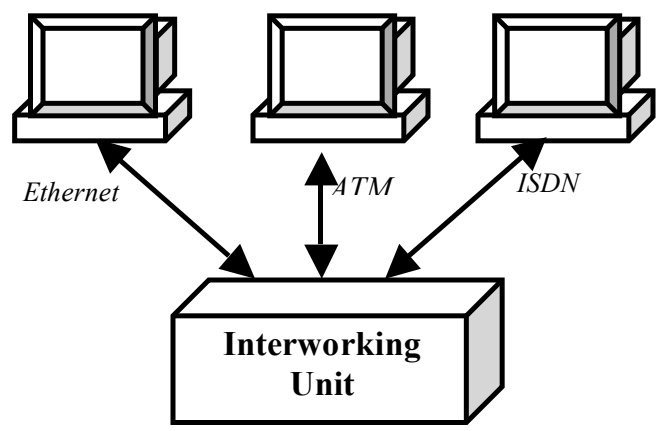

Figure 1: - Interconnection of ISDN ATM and Ethernet Terminals through the Interworking Unit. 
While each line interface module performs the line termination and the associated transfer protocols for each network environment, the processor performs the protocol conversion between different environments.

The proposed Interworking device has two data buses: the Utopia Bus [1], which sustains the cell transfer between line interface modules; and the processor bus, which allows that ATM cells, ISDN channels and Ethernet packets, maybe transferred between line interfaces, the processor, and the memory.

The processor unit, based on the Motorola PowerQuicc [2] architecture and the Tornado operation system, is able to support real time applications.

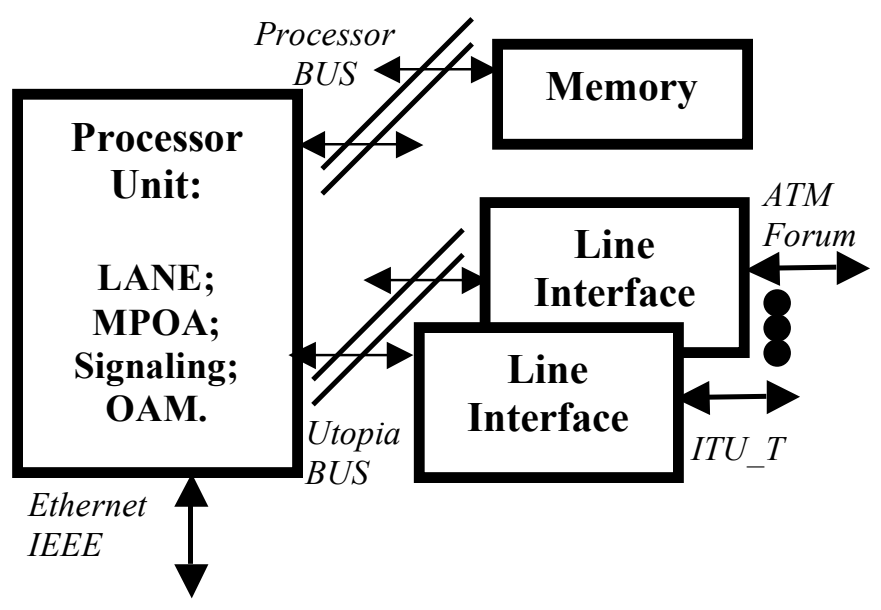

Figure 2: - Hardware Architecture of de Interworking Unit.

The adaptation of data formats and protocols are implemented at the ATM Adaptation Layer (AAL) [3], by mapping the Ethernet packets or ISDN stream into ATM cells, which are transferred on ATM Virtual Path or Virtual Channel Connections (VPC/VCC), over switched or semipermanent circuits, according to ITU specifications [4].

This processor unit also has capacity, not only to dial with different data formats and protocols of the several network environments, but also to support ATM and ISDN signaling, together with LAN Emulation (LANE) [5] and Multiprotocol Over ATM (MPOA) [6] procedures.

\section{PhySICAL AND LOGICAL INTERFACES}

Since the IWU have to convert different network environments, the proposed electronic device has to support the appropriated physical and logical interfaces to interconnect several types of terminals.

\section{A. ISDN Interfaces}

The Interworking Unit has two ISDN interfaces for the basic and the primary access, respectively.

1) Basic Access

The Basic Access Interface is specified at the reference point S, by several ITU recommendations [7].

With a transmission rate of $144 \mathrm{Kbit} / \mathrm{s}, \mathrm{S}$ bus is able to interconnected at least 8 terminals, by sharing one or two 64 $\mathrm{Kbit} / \mathrm{s}$ time slots for data information transfer (B channel), while the signaling information is transported out of band on a $16 \mathrm{kbit} / \mathrm{s}$ D Channel.

The Basic Access Interface can be used to interconnect ISDN terminals to Local Area Networks (LANs) over a Public Switched Network (PSN), trough one or more of the other interfaces of the IWU (i.e. the Primary ISDN access, the ATM interface or the Ethernet).

\section{2) Primary Access}

Physical and logical characteristics of ISDN primary access interface are specified by ITU [8].

While the basic access interface is able to interconnect ISDN terminals trough different LANs over the PSN, the primary access interface is appropriate to support the interconnection of different network environment, such as ATM or Ethernet.

\section{B. Ethernet Interface}

Since most of computer terminals communicate trough an Ethernet Interface, according to IEEE 802.3 standards, namely 10 Base $\mathrm{T}$ and 100 Base $\mathrm{T}$ specifications, the proposed IWU have to handle with these interfaces at physical and logical levels, to convert the Ethernet environment to ISDN or ATM specifications.

\section{ATM Interfaces}

The Interworking Unit has two ATM physical interfaces: one operates at $2 \mathrm{Mbit} / \mathrm{s}$ and the other operates at $25 \mathrm{Mbit} / \mathrm{s}$.

\section{1) $I T U-T G 804$}

On the $2 \mathrm{MBit} / \mathrm{s}$ ATM interface, the cells are mapped according ITU-T recommendation G. 804 [9] on the frame described by G. 703 [8]. This interface is appropriate to the interconnection of ISDN and ATM environments over the PSN, or to interconnect LANs trough ATM networks. 


\section{2) ATM Forum $25.6 \mathrm{Mbit} / \mathrm{s}$}

The ATM Forum 25,6 Mbit/s over twisted pair interface [10] is suitable to support the interconnection of ATM terminals to the ATM or LANE Networks.

\section{INTERCONNECTION CENARIOS}

Most of standardised ISDN primary access interfaces, such as Wired, Optical or RF Links, as well as, Add and Drop Wired Rings, operating at $2 \mathrm{Mbit} / \mathrm{s}$, are able to interconnect remotely the Interworking device to one PSN Local Exchange or to another IWU. Other standardised interfaces, such as the ISDN basic access, the 25,6 Mbit/s and the Ethernet are more suitable for terminal interconnections, within ISDN, ATM or Ethernet environments.

The figure 3 sketches the interconnection of the IWU with ISDN, ATM or Ethernet networks, to perform remotely the interworking procedures between different type of terminals.

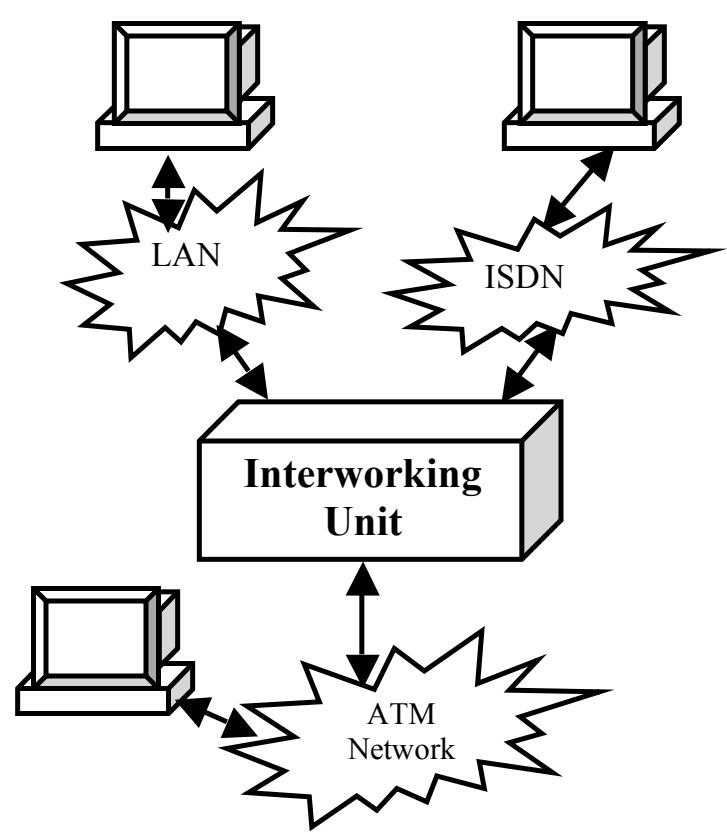

Figure 3: - Interworking between ISDN, LAN ant ATM Networks.

The IWU have to dial to two main interworking scenarios: ATM/ISDN and ATM/Ethernet. In both cases, Management, as well as Operation and Maintenance (OAM) procedures, carry out by appropriated protocols [11], are also supported by the IWU.

\section{A. ATM/ISDN Interworking}

The bloc diagram of figure 4 presents the logical architecture of the IWU for the ISDN and ATM services conversion. For this propose, the IWU has to convert data formats between ATM and ISDN interfaces, as well as the signaling and management protocols, according the ITU specifications [12].

The information flow of ISDN services supported by HighLevel Dada Link Control (HDLC) protocols, such as the Link Access Procedure Balanced (LAPB), the Link Access Procedure on D Channel (LAPD) and the Link Access Procedure for Frame-Mode Bearer Services (LAPF), are converting to ATM formats, trough the ATM Adaptation Layer (AAL) type 1 or type 3/4 [3], and are supported by ATM Layer Virtual Path or Virtual Channel Connections (VPC/VCC) [4]. The signaling information related to ISDN and ATM services is also converted on the IWU, according to ITU-T Q.931 and ATM Forum UNI specifications [13].

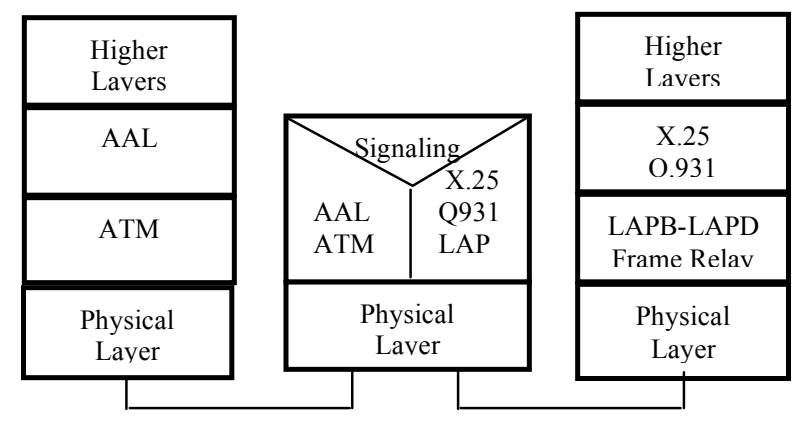

Figure 4: - Logical Architecture of the Interworking Unit for ISDN services.

\section{B. ATM/Ethernet Interworking}

The proposed IWU supports Routing, Bridge and Gateway functions on ATM/Ethernet environments. The conversion of ATM and Ethernet traffic formats and protocols, is made according the LAN Emulation specifications [5].

The IWU implements the LAN Emulation Sever (LES), which supports the ATM Address Resolution Protocol (ATMARP), the LAN Emulation User-Network Interface (LUNI) and the Interim Local Management Interface (ILMI). Figure 5 presents the IWU logical architecture for LAN Emulation over ATM, according the ATM Forum specifications.

The IWU also support other LAN Emulation functionalities, such as Broadcast and Unknown Sever (BUS) and LAN Emulation Configuration Sever (LECS), to permit that ATM terminals, supporting the LAN Emulation Client 
(LEC) functions, may communicate with other Ethernet terminals, without the intervention of external equipment.

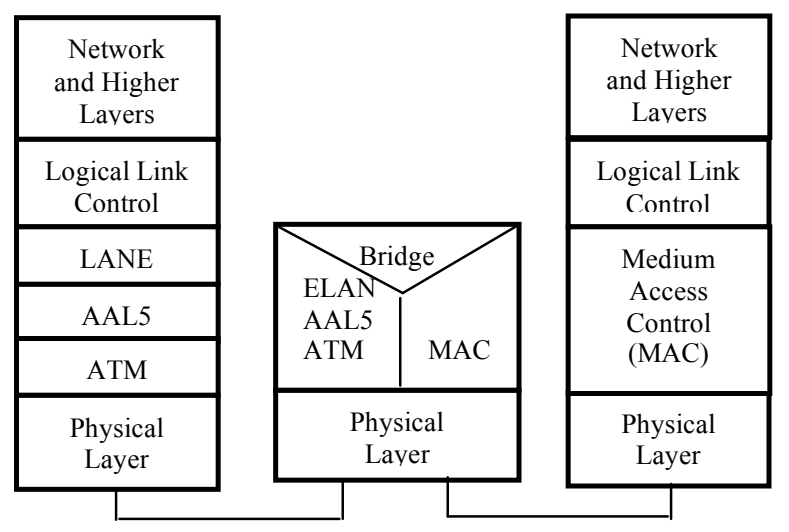

Figure 5: - Logical Architecture of the Interworking Unit for LAN Emulation.

Other standardized protocols, such as the Classical IP over ATM (IPOA) and the Multi-Protocol Over ATM (MPOA) [6] could also be implemented in the future, together with the Next Hop Resolution Protocol (NHRP) and the Next Hop Resolution Protocol Sever (NHS).

Broadcast and multicast capabilities could also be supported with the implementation of Multicast Sever (MCS) and Multicast Address Resolution Sever (MARS) functionalities.

\section{Management Procedures}

The Interworking Unit carries out as well management procedures, supported by SNMP, CNM [14] or TMN protocols. In addition, Operation and Maintenance (OAM) procedures for ATM environment [11] are used to control, in a very flexibly way, some hardware capabilities, namely the remote configuration to execute all the possible combination of the interworking functions between ATM, ISDN and Ethernet protocols.

\section{CONCLUSIONS}

In our days, the Asynchronous Transfer Mode (ATM) establishes, more than, the basis structure of the Broadband Integrated Services Digital Network (B-ISDN), a shared platform for the transmission and the switching over most of circuit or packet oriented networks.

The Interworking Unit, presented on this paper, has a flexible architecture, based on a central processor unit, which control the information flux between the modular line interface broads through two independent data buses, and is able to processes, in real time, the protocol conversion between the involved data formats. This electronic device can be integrated on a wide verity of communication infrastructures and traffic environments, for interconnection of ATM, ISDN or LAN terminals, locally or remotely, over a public telecommunication network.

\section{REFERENCES}

[1] ATM Forum, " Utopia 3 Physical Layer Interface", ftp://ftp.atmforum.com/pub/approved-specs/af-phy-0136.000.pdf, Nov. 1999.

[2] Motorola, MPC860: PowerQuicc - Integrated PowerPC Microprocessor, http://ebus.motsps.com/ProdCat/psp/0,1250,MPC860 M98655,00.html.

[3] ITU-T, "B-ISDN ATM Adaptation Layer (AAL) specification", Rec. I.363, Geneva, 1993.

[4] ITU-T, "B-ISDN ATM Layer Specification", Rec. I.361, Geneva, 1995.

[5] ATM Forum, "LAN Emulation over ATM Version 2 - LNNI Specification", ftp://ftp.atmforum.com/pub/approved-specs/af-lane0112.000.pdf, Feb. 1999.

[6] ATM Forum, "Multi-Protocol Over ATM Specification v1.0", ftp://ftp.atmforum.com/pub/approved-specs/af-mpoa-0087.000.pdf, July, 1997.

[7] ITU-T, " Integrated services digital networks (ISDN)", Rec. I.120, Geneva, March 1993.

[8] CCITT, "General Aspects of Digital Transmission Systems; Terminal Equipements", Blue Book - Rec. G.700 - G.772, Vol. III-4. CCITT, Geneva, 1989

[9] I TU-T, "ATM cell mapping into plesiochronous digital hierarchy (PDH)", Rec. G.804, Geneva, 1998.

[10] ATM Forum, "Physical Interface Specification for $25.6 \mathrm{Mb} / \mathrm{s}$ over Twisted Pair", ftp://ftp.atmforum.com/pub/approved-specs/afphy-0040.000.pdf, Nov. 1995.

[11] ITU-T, "B-ISDN Operation and Maintenance principles and functions", Rec. I.610, Geneva, 1995.

[12] ITU-T, "B-ISDN Protocol Reference Model and its Application", Rec. I.321, Geneva, 1991.

[13] ATM Forum, " UNI Signaling 4.0", ftp://ftp.atmforum.com/pub/approved-specs/af-sig-0061.000.pdf, Jul. 1996.

[14] ATM Forum, " Customer Network Management (CNM) for ATM Public Network Service", ftp://ftp.atmforum.com/pub/approved-specs/af-nm-0019.000.pdf, Oct. 1994. 